\title{
Non-Discriminant Relationships between Leg Muscle Strength, Mass and Gait Performance in Healthy Young and Old Adults
}

\author{
Thomas Muehlbauer ${ }^{a}$ b $\quad$ Urs Granacher ${ }^{a}$ Ron Borde ${ }^{a}$ Tibor Hortobágyi ${ }^{c}$ \\ a Division of Training and Movement Sciences, Research Focus Cognition Sciences, University of Potsdam, Potsdam, \\ and ${ }^{\mathrm{b}}$ Division of Movement and Training Sciences/Biomechanics of Sport, University of Duisburg-Essen, Essen, \\ Germany; ${ }^{C}$ Center for Human Movement Sciences, University Medical Center Groningen, Groningen, Netherlands
}

\section{Keywords}

Body composition $\cdot$ Muscle torque $\cdot$ Walking $\cdot$ Seniors

\begin{abstract}
Background: Gait speed declines with increasing age, but it is unclear if gait speed preferentially correlates with leg muscle strength or mass. Objective: We determined the relationship between gait speed and (1) leg muscle strength measured at 3 lower extremity joints and (2) leg lean tissue mass (LTM) in healthy young (age: 25 years, $n=20$ ) and old (age: 70 years, $n=20$ ) adults. Methods: Subjects were tested for maximal isokinetic hip, knee, and ankle extension torque, leg LTM by bioimpedance, and gait performance (i.e., gait speed, stride length) at preferred and maximal gait speeds. Results: We found no evidence for a preferential relationship between gait performance and leg muscle strength compared with gait performance and leg LTM in healthy young and old adults. In old adults, hip extensor strength only predicted habitual gait speed $\left(R^{2}=0.29, p=0.015\right)$, whereas ankle plantarflexion strength only predicted maximal gait speed and stride length (both $R^{2}=0.40, p=0.003$ ). Conclusions: Gait speed did not preferentially correlate with leg muscle strength or leg LTM, favoring neither outcome for predicting mobility. Thus, we recommend that both leg muscle strength and leg LTM should be tested and trained complementarily.
\end{abstract}

\section{KARGER}

(C) 2017 S. Karger AG, Basel

E-Mail karger@karger.com

www.karger.com/ger
Further, hip and ankle extension torque predicted gait performance, and thus we recommend to test and train healthy old adults by functional integrated multiarticular rather than monoarticular lower extremity strength exercises.

(c) 2017 S. Karger AG, Basel

\section{Introduction}

Gait speed, a marker of mobility, declines with increasing old age, and slow gait is associated with adverse clinical and cognitive conditions [1]. An understanding of factors, including muscle strength and mass/size, underlying the evolution of slow gait is thus clinically relevant. A loss of alpha motoneurons and reductions in the number and size of type 2 muscle fibers in particular contribute to the age-related decreases in muscle strength and size $[2,3]$. Although losses in muscle strength and size correlate [4], longitudinal studies $[2,5]$ that investigated 9 to 12 [5] and 1,678 [2] old adults reported a 1.5-5 times greater decline in lower extremity muscle strength than muscle size. This raises the possibility that leg muscle strength and leg muscle mass are differently related to gait speed.

The age-related strength loss is not uniform between lower extremity joints. The rate of decline in ankle plantarflexor force is the largest compared with other leg mus-

Prof. Thomas Muehlbauer, $\mathrm{PhD}$

Division of Movement and Training Sciences/Biomechanics of Sport University of Duisburg-Essen

Gladbecker Strasse 182, DE-45141 Essen (Germany)

E-Mail thomas.muehlbauer@uni-due.de 
cle groups [6], reaching 4 times greater loss than the dorsiflexors over a 12-year period before the age 97 [7]. There are also preferential reductions of $\sim 25 \%$ in type 2 fiber area and mitochondrial and citrate synthase enzyme activities in old compared with young adults' gastrocnemius $[8,9]$. The behavioral manifestation of such changes in ankle plantarflexor muscle strength and size is the age-related reduction in ankle and the compensatory increase in hip joint torques and power outcomes measured during gait $[10,11]$. Thus, the contribution of ankle plantarflexors and hip extensors to old adults' gait speed decreases and increases, respectively. However, predictions of gait speed from lower extremity joint-specific muscle strength revealed opposing results $[12,13]$. In view of the biomechanical joint kinetics data, it is conceivable that the relationship between gait speed and muscle strength differs at the different lower extremity joints. Because of the diminished and increased contribution, respectively, by the ankle plantarflexors and hip extensors to the total mechanical output during gait [11], we expect to find different correlations between gait speed and ankle plantarflexor (low correlation) versus hip extensor (high correlations) maximal voluntary torques in old adults. Such an expectation contrasts with the prevailing data that focused on the relationship between mobility and knee extensor function $[10,14]$ even though old age has virtually no effects on knee kinetics measured during gait $[10,11]$.

Because of the heterogeneous reductions in leg muscle strength and size and the low association between leg muscle mass and strength, it is clinically relevant to determine the relationship between gait speed and leg muscle mass. The sporadic data are inconsistent, as knee extensor muscle thickness did not correlate $(r=-0.07)$ with gait speed in women aged 52-83 years [15], whereas bone-free lean tissue mass (LTM) in the legs revealed a low but significant $(r=-0.12, p \leq 0.01)$ correlation with gait speed in older adults aged $68 \pm 9$ years [16]. In addition, only small associations were detected between changes in leg muscle mass and changes in gait speed in 66- to 85-year-old female athletes [17], in 25 resistancetrained women aged 71 years [18], after a diet-exercise intervention in healthy women aged $\sim 60$ years [19], and after resistance training in 23 healthy women aged 70 years [20]. Because of the disproportionately larger reductions in leg muscle strength compared with leg muscle mass [21], it is conceivable that the relationship between gait performance and leg muscle strength are stronger than between gait performance and leg muscle mass.

The purpose of the study was to determine the relationship between gait speed and (1) leg muscle strength measured at 3 lower extremity joints and (2) leg LTM in healthy young and old adults. We expected to find stronger associations between leg muscle strength, in particular, hip extension strength and gait speed compared with leg LTM and gait speed in old but less so in young adults. Notably, this should be observed when maximum physiological capacity is required, i.e. during walking at fast speed. The findings would guide clinicians concerning the choice between leg muscle strength and leg LTM as predictors of healthy old adults' mobility. In addition, results from this study could be used to design resistance training programs with the goal to prevent mobility disability in seniors.

\section{Methods}

\section{Participants}

Healthy young $(n=20)$ and old $(n=20)$ adults were recruited from the local community through advertisements in regional newspapers. Participants were without cognitive impairment, currently free of, and had no history of musculoskeletal, neurological, or orthopedic disorders. The local ethics committee approved the study protocol and the consent form, signed by each participant before the start of the study conducted according to the latest version of the Declaration of Helsinki.

\section{Testing Procedure}

Participants completed the Lateral Preference Inventory (LPI), the Freiburg questionnaire for everyday and sports-related activities (FQoPA), the Mini Mental State Examination (MMSE), and the Clock Drawing Test (CDT). Participants received standardized verbal instructions, watched visual demonstrations of the experimental procedures, and were familiarized with each testing device and protocol. After warming up by riding a bicycle ergometer for $10 \mathrm{~min}$ at a rate of perceived exertion of 12 on the 6-20 Borg scale, participants were assessed for: (a) leg LTM, (b) 10-m walk at preferred and maximal speed, and (c) maximal isokinetic torque (MIT) of the hip, knee, and ankle extensors (Fig. 1). This testing sequence was applied to minimize the effects of neuromuscular fatigue.

\section{Assessment of Leg LTM}

We assessed body composition by noninvasive bioelectrical impedance analysis (BIA) (Fig. 1). Participants abstained from caffeine and alcohol for $24 \mathrm{~h}$, and exercise for $12 \mathrm{~h}$ prior to testing. The octopolar tactile-electrode impedance meter estimated body composition at $5,50,250,500 \mathrm{kHz}$, and $1 \mathrm{MHz}$ under laboratory conditions while standing barefoot on the device (InBody 720, BioSpace, Seoul, Korea). This method was validated by dual-energy X-ray absorptiometry (DEXA) $\left(R^{2}=0.93\right)$ and the muscle mass data revealed excellent agreement with DEXA in adults (ICC values in males and females amounted to 0.96 and 0.95 , respectively) [22]. Leg LTM of BIA measured with the InBody 720 correlates with leg skeletal muscle mass measured with DEXA $\left(R^{2} \geq 0.79\right)$ [23]. We used the dominant leg's LTM in the analyses.
12

Gerontology 2018;64:11-18

DOI: $10.1159 / 000480150$
Muehlbauer/Granacher/Borde/ Hortobágyi 


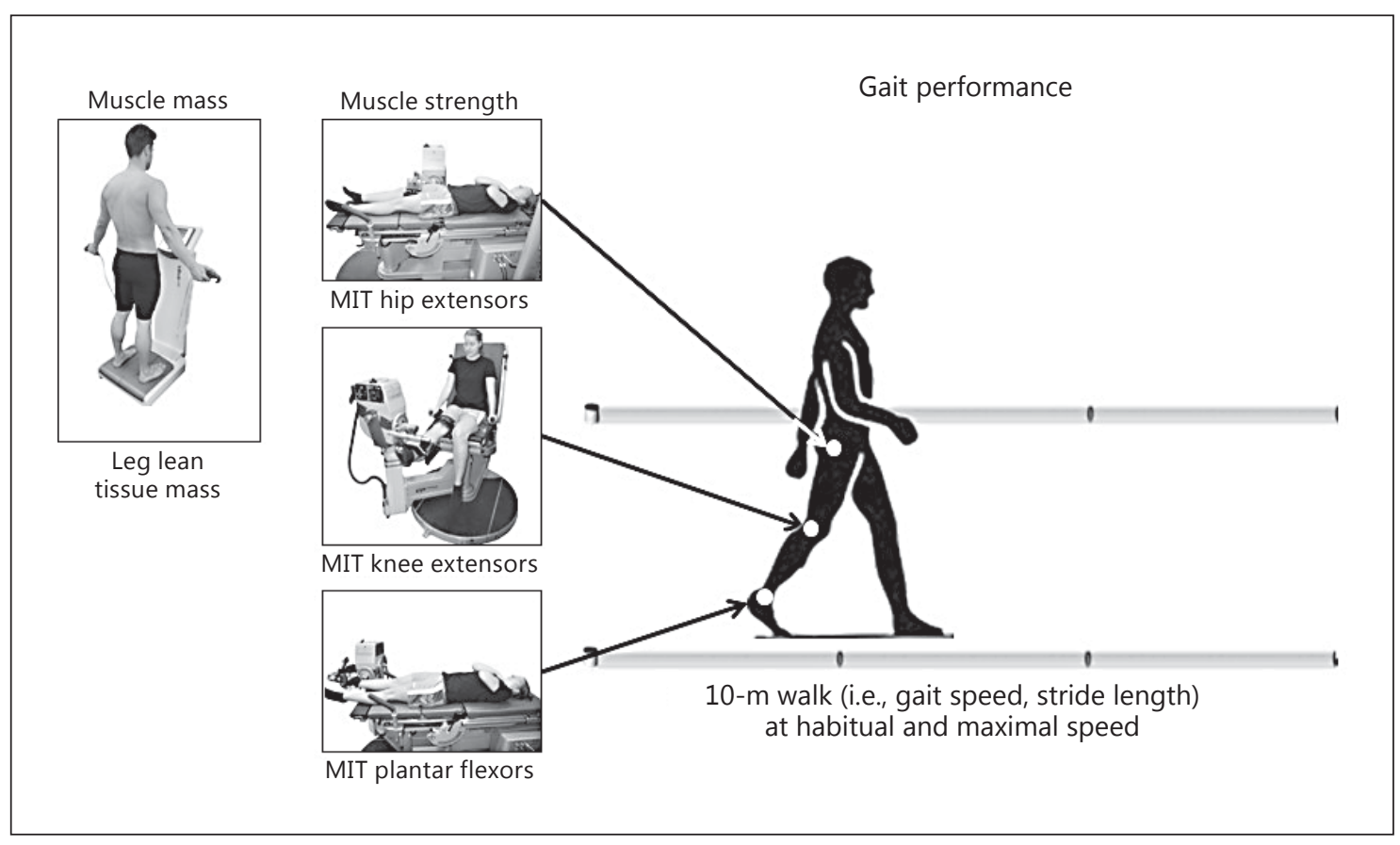

Fig. 1. Experimental setup. MIT, maximal isokinetic torque.

\section{Assessment of Maximal Isokinetic Torque}

MIT of the hip, knee, and ankle extensors of the dominant leg was measured on an isokinetic dynamometer (Isomed $2000^{\circledR}$, D\&R Ferstl GmbH, Hemau, Germany). The maximum error of the torque sensor was $<0.2 \%$. During hip extension, participants lay supine on the padded seat of the dynamometer. A padded attachment was strapped around the thigh of the tested limb and belts stabilized the pelvis and trunk. Participants crossed their arms in front of the chest. Range of motion of the hip was limited to $5^{\circ}$ of extension and to $90^{\circ}$ of flexion to minimize overstretching in old adults. The approximate axis of the hip joint (i.e., greater trochanter) was aligned with the dynamometer's axis of rotation [12]. The initial position of the leg was an almost full extension of the hip and knee joint. Torque was assessed during hip extension movements starting at $90^{\circ}$ hip flexion to full extension (Fig. 1). Isokinetic knee extension torque was measured in a sitting position with the hip in $90^{\circ}$ flexion. The thigh was firmly strapped to the dynamometer's seat and subjects grasped the handles on either side of the seat. The padded cuff was placed on the tibia, $5 \mathrm{~cm}$ proximal to lateral malleolus. The range of motion was set to $15^{\circ}$ of flexion and $75^{\circ}$ of extension. The center of the knee joint was aligned with the dynamometer's axis of rotation. Measurements started in full flexion and ended in full extension (Fig. 1). For the plantarflexion test, participants lay supine on the dynamometer's seat, with hip and knee joints in full extension. Straps stabilized the shoulders, waist, thigh, shank, and the foot to minimize extraneous upper body movements. Arms were folded in front of the chest. Plantarflexion MIT was measured between $10^{\circ}$ of dorsiflexion and $30^{\circ}$ of plantarflexion, starting the movement in full dorsiflexion. The malloleus lateralis was aligned with the dynamometer's axis of rotation and the foot strapped to the footplate (Fig. 1).

Leg Muscle Strength and Mass Correlates of Gait Performance
The order of leg joint testing was randomized. Participants performed 5 submaximal warm-up trials to get accustomed to the testing procedure and performed 5 maximal effort trials in terms of force and speed at $60 \%$ s for each joint. Participants were instructed to "act as powerfully and as fast as possible." No verbal encouragement was provided. The torque signal was sampled at $200 \mathrm{~Hz}$ and filtered by a digital 4th order recursive Butterworth low-pass filter with a cutoff frequency of $50 \mathrm{~Hz}$. In offline analyses of the torque-time curves, the trial with the maximal value in terms of MIT was determined. MIT values were normalized for body mass and height $(\mathrm{Nm} / \mathrm{kg} \times \mathrm{m})$ and included in our data analyses.

\section{Assessment of Gait Performance}

We measured gait on a 10-m-long, optoelectrical walkway instrumented with light-emitting diodes bars and sensors, sampled at $1 \mathrm{kHz}$ that can detect key gait events (OptoGait, Bolzano, Italy; Fig. 1). Participants walked in their personal footwear at preferred and maximal speed, initiating and terminating each walk a minimum of $2 \mathrm{~m}$ before and after the 10-m walkway. For the fast walking condition, participants were instructed to "walk as fast as you can without running and without feeling that you will trip or fall." One walking trial was performed for each gait speed condition. The OptoGait system has high discriminant and concurrent validity with a validated electronic walkway (GAITRite system) for the assessment of spatiotemporal gait parameters in adults [24]. Stride length $(\mathrm{cm})$ was defined as the linear distance between successive heel contacts of the same foot and gait speed as stride length divided by stride time $(\mathrm{m} / \mathrm{s})$. 
Table 1. Characteristics of participants by age group

\begin{tabular}{lllll}
\hline & Young adults $(n=20)$ & Old adults $(n=20)$ & $t$ value & $p$ value \\
\hline Age, years & $25.0(2.9)$ & $70.1(3.8)$ & & \\
Sex, F/M & $10 / 10$ & $10 / 10$ & 0.78 & 0.440 \\
Body mass, kg & $72.4(13.5)$ & $69.4(10.7)$ & 3.76 & 0.001 \\
Body height, cm & $177.5(7.8)$ & $167.9(8.4)$ & 1.76 & 0.086 \\
BMI, kg/m & $22.9(2.9)$ & $24.6(3.5)$ & & 0.856 \\
CDT & & normal & 0.18 & 0.332 \\
MMSE, points & $14.2(9.5)$ & $28.8(0.9)$ & 0.98 & \\
PA, h/week & $82.8(4.5)$ & $81.4(4.7)$ & & \\
Leg length ${ }^{\text {a }}$ cm & $0 / 20$ & $0 / 20$ & & \\
Leg preference, L/R & & & & \\
\end{tabular}

Values are means $( \pm S D)$. BMI, body mass index; CDT, Clock drawing test; MMSE, Mini-Mental State Examination; PA, physical activity; F, female; M, male; L, left; R, right. ${ }^{\text {a }}$ Measured from the trochanter major to the malleolus lateralis.

\begin{abstract}
Statistical Analysis
Data are presented as mean and standard deviation. A multivariate analysis of variance was used to detect differences between young and old adults in all examined variables. Cohen's $d$ was calculated to determine whether a statistical difference was practically meaningful as small $(0 \leq d \leq 0.49)$, medium $(0.50 \leq d \leq 0.79)$, and large $(d \geq 0.80)$. Pearson product-moment correlation coefficients were computed to calculate the associations between leg muscle strength, leg LTM and gait performance, and values of $0 \leq r$ $\leq 0.69$ indicated a small, $0.70 \leq r \leq 0.89$ a medium, and $r \geq 0.90$ a large correlation [25]. We compared correlation coefficients with Fisher $z$-transformation. As in former studies $[12,13]$, a forwardmoving linear stepwise regression analysis identified the predictors of gait performance. All analyses were done in the Statistical Package for Social Sciences (SPSS) version 22.0, and significance level was set at $p<0.05$.
\end{abstract}

\section{Results}

Age Differences in Leg Muscle Strength, Leg LTM, and Gait Performance

Table 1 shows that participants were physically and mentally healthy, normal weight, and physically active. In young adults, males showed significantly higher body mass, body height, and BMI compared to females. In old adults, we detected significantly higher body mass and body height in males compared to females. No sex-specific differences were found for BMI. Table 2 shows that old versus young adults had 20\% lower leg LTM and 33$37 \%$ lower joint-specific muscle strength; this is $34 \%$ lower summed leg muscle strength (all $p<0.05$ ). There was a trend for ankle versus hip MIT being 4\% lower. Old ver- sus young adults walked 7 and $17 \%$ slower at preferred and maximal speed, respectively, using 6 and $15 \%$ shorter strides (all $p<0.05)$.

Associations between Leg Muscle Strength, Leg LTM, and Gait Performance

Leg muscle strength, leg LTM, and gait performance did not correlate in young adults (data not shown). In old adults, Table 3 shows that preferred gait speed did not significantly correlate with leg LTM and any of the strength measures (range $r=0.05-0.40$, all $p>0.05$ ). Stride length correlated with leg LTM $(r=0.59, p<0.01)$ and 3 of 4 measures of leg strength (range $r=0.47$ to 0.55 , all $p<0.05$ ). In addition, Table 3 shows that 8 of 10 correlations were higher at maximal versus preferred gait speed. Compared with leg LTM, maximal gait speed correlated more strongly with MIT independent of joint considered (range $r=0.54-0.66$, all $p<0.05$ ). Leg LTM correlated with stride length $(r=0.59, p<0.01)$ and the correlations of MIT with stride length and MIT with gait speed were quite similar (range $r=0.52-0.63$, all $p<0.05$ ). Figure 2 shows the linear regression of leg LTM and leg torque on maximal gait speed in old adults.

A comparison of correlations between leg LTM and gait performance (i.e., gait speed, stride length) and jointspecific leg muscle strength and gait performance revealed no statistical differences (range Fisher $z=0.18$ 1.17 , all $p>0.05$ ). There were also no significant differences in the magnitude of correlations between stride length and gait speed and hip, knee, and ankle MIT (range Fisher $z=0.05-1.47$, all $p>0.05$ ).
$14 \quad$ Gerontology 2018;64:11-18 DOI: $10.1159 / 000480150$
Muehlbauer/Granacher/Borde/ Hortobágyi 
Table 2. Leg LTM, strength, and gait performance by age group

\begin{tabular}{|c|c|c|c|c|c|c|}
\hline & $\begin{array}{l}\text { Young adults } \\
(n=20)\end{array}$ & $\begin{array}{l}\text { Old adults } \\
(n=20)\end{array}$ & $\Delta, \%$ & $t$ value & $p$ value & Cohen's $d$ \\
\hline \multicolumn{7}{|l|}{ Leg muscle mass, $\mathrm{kg}$} \\
\hline Leg LTM & $9.6(1.8)$ & $7.7(1.5)$ & 20 & 3.53 & 0.001 & 2.24 \\
\hline \multicolumn{7}{|c|}{ Leg muscle torque, $\mathrm{Nm} / \mathrm{kg} \times \mathrm{m}$} \\
\hline Hip & $1.5(0.5)$ & $1.0(0.4)$ & 33 & 3.30 & 0.002 & 2.08 \\
\hline Knee & $1.2(0.3)$ & $0.8(0.2)$ & 33 & 5.61 & $<0.001$ & 3.54 \\
\hline Ankle & $0.8(0.2)$ & $0.5(0.2)$ & 37 & 4.99 & $<0.001$ & 3.16 \\
\hline Summed & $3.5(0.9)$ & $2.3(0.7)$ & 34 & 4.90 & $<0.001$ & 3.10 \\
\hline \multicolumn{7}{|c|}{ Gait at preferred speed } \\
\hline Gait speed, m/s & $1.6(0.1)$ & $1.5(0.1)$ & 7 & 2.19 & 0.035 & 1.38 \\
\hline Stride length, cm & $156.7(11.8)$ & $147.3(11.9)$ & 6 & 2.50 & 0.017 & 1.58 \\
\hline \multicolumn{7}{|c|}{ Gait at maximal speed } \\
\hline Gait speed, m/s & $2.4(0.3)$ & $2.0(0.3)$ & 17 & 4.19 & $<0.001$ & 2.66 \\
\hline Stride length, cm & $194.6(16.6)$ & $170.6(18.1)$ & 12 & 4.37 & $<0.001$ & 2.76 \\
\hline
\end{tabular}

Values are means $( \pm \mathrm{SD})$. LTM, lean tissue mass; summed, sum of hip, knee, and ankle maximal isokinetic torque.

A forward-moving linear stepwise regression showed that hip extensor MIT only predicted stride length $\left(R^{2}=\right.$ $0.29, p=0.015)$ during preferred walking and ankle plantarflexor MIT only predicted stride length $\left(R^{2}=0.40, p=\right.$ $0.003)$ and gait speed $\left(R^{2}=0.40, p=0.003\right)$ during walking at maximal speed (Table 4 ).

\section{Discussion}

Against the hypothesis, we found no evidence for a preferential relationship between gait performance and leg muscle strength compared with gait performance and leg LTM in either age group. Thus, we recommend that both leg muscle strength and leg LTM should be tested and trained complementarily. At preferred gait speed, there was some evidence for the hypothesis that hip extensor strength only predicted gait performance, whereas ankle plantarflexion strength only predicted old adults' maximal gait speed and stride length. On balance, we recommend multiarticular rather than monoarticular testing and training of the lower extremities in old adults.

The present analyses are based on data from 20 healthy young adults with age-normal characteristics and 20, 70-year-old adults with typical body mass $(69.4 \mathrm{~kg})$, BMI $\left(24.6 \mathrm{~kg} / \mathrm{m}^{2}\right)$, and high daily physical activity $(\sim 2 \mathrm{~h} /$ day $)$ without apparent comorbidities (Table 1). Our old adults' $1.5 \mathrm{~m} / \mathrm{s}$ habitual gait speed is at the high end of habitual

Leg Muscle Strength and Mass Correlates of Gait Performance
Table 3. Association of leg LTM, strength, and gait performance in old adults by gait speed condition

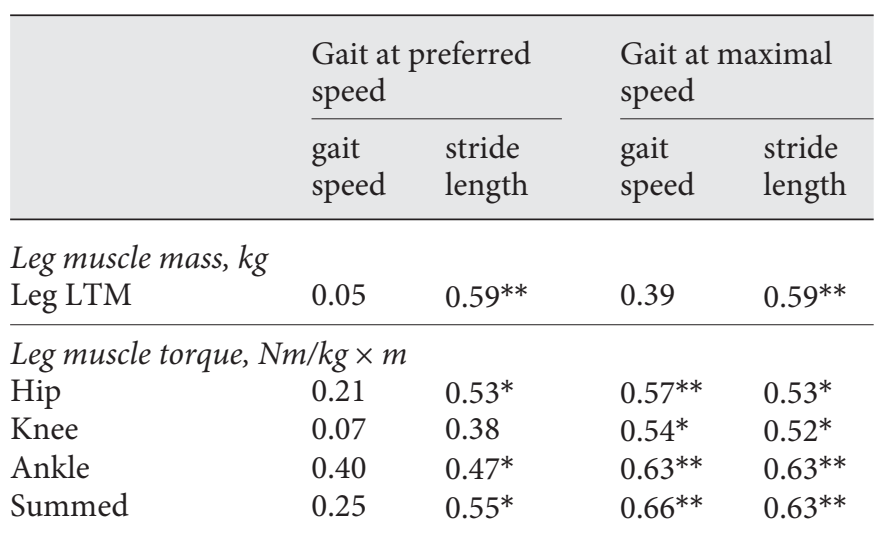

LTM, lean tissue mass; summed, sum of hip, knee, and ankle maximal isokinetic torque. ${ }^{*} p<0.05 ;{ }^{* *} p<0.01$.

gait speed norms of 1.15 [26], 1.24 [14], and $1.30 \mathrm{~m} / \mathrm{s}$ [27] as is the $2.0 \mathrm{~m} / \mathrm{s}$ maximal gait speed (normal range: $1.44-$ $1.99 \mathrm{~m} / \mathrm{s}$ ). Still, relative to the reference young adults group, old adults walked $\sim 12 \%$ slower with $\sim 11 \%$ shorter steps, had $18 \%$ lower leg LTM and were $34 \%$ weaker (all $p<0.05$, Table 2). The lower leg LTM data are in line with the previously reported $15 \%$ difference between young and 71- to 87-year-old adults [28], as are the joint-

Gerontology 2018;64:11-18 15 
Fig. 2. Regression of leg lean tissue mass (a) and summed ankle, knee, and hip extension maximal voluntary torque measured at $60 \%$ and normalized for body mass and height (b) on gait speed in healthy old adults $(n=20)$. The regression is described by the equation of $y=0.07 \mathrm{x}+1.5, R^{2}=0.15$ $(p>0.05 ; \mathbf{a})$ and $y=0.25 \mathrm{x}+1.4, R^{2}=0.44$ $(p<0.05 ; \mathbf{b})$.

Table 4. Results of stepwise regression analyses for measures of leg muscle strength and gait performance in old adults by gait speed condition

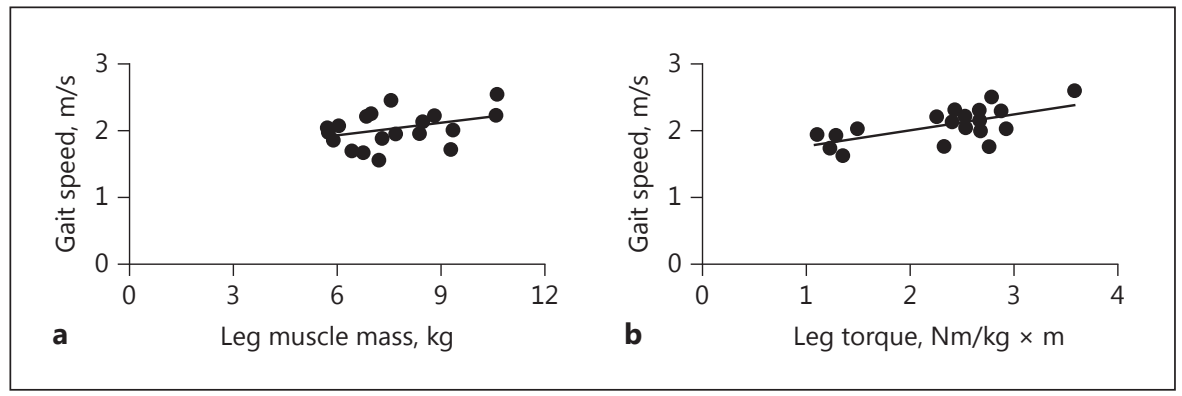

\begin{tabular}{|c|c|c|c|c|c|c|c|c|}
\hline & \multicolumn{4}{|c|}{ Gait at preferred speed } & \multicolumn{4}{|c|}{ Gait at maximal speed } \\
\hline & $\beta$ & SE & $p$ value & $R^{2}$ & $\beta$ & SE & $p$ value & $R^{2}$ \\
\hline $\begin{array}{l}\text { Gait speed } \\
\text { MIT ankle }\end{array}$ & & & & & 0.630 & 0.257 & 0.003 & 0.40 \\
\hline $\begin{array}{l}\text { Stride length } \\
\text { MIT hip } \\
\text { MIT ankle }\end{array}$ & 0.534 & 6.056 & 0.015 & 0.29 & 0.629 & 18.518 & 0.003 & 0.40 \\
\hline
\end{tabular}

$\beta$, parameter estimate; $R^{2}$, proportion of variance explained by the regression model; MIT, maximal isokinetic torque; SE, standard error. specific and summed MIT values [6], confirming the substantially greater reductions in leg muscle strength than leg LTM [29]. Thus, our analyses are relevant to a sample of cognitively and physically healthy old adults, showing signs of normal aging.

Associations between Leg Muscle Strength, Leg LTM, and Gait Performance

Longitudinal $[2,5]$ studies reported 1.5 times greater loss of leg muscle strength than leg muscle size, raising the possibility of a stronger relationship between leg muscle strength versus leg muscle size and gait performance. However, our data did not bear out this expectation at either gait speed, confirming previous findings of similar correlations [16]. The non-discriminant predictive power of these two measures may be related to old adults being well above the $1.0 \mathrm{~m} / \mathrm{s}$ clinical threshold of habitual gait speed and well below a function-affecting threshold of leg muscle strength loss [10]. But even when below the threshold, i.e. gait speed of $0.8 \mathrm{~m} / \mathrm{s}$, a $0.17-\mathrm{kg}$ greater dietinduced increase in muscle mass versus control did not improve gait speed ( $p=0.46$ for between-group differences) and performance in a composite mobility test ( $p=$ 0.51) [30]. While muscle power compared with leg strength may be functionally more relevant to gait speed [31], at 3-year follow-up, declines of muscle size, strength, power, and scores in a composite mobility test were similar [32]. In addition, leg press power correlated with functional status as weakly $(r=0.64, p<0.001)$ [33] as muscle strength correlated with gait speed in the present study (range $r=0.55-0.63, p<0.05$; Table 2, Fig. 2b). While the age-related loss of leg power can exceed that of leg strength [34], curiously the force compared with the velocity component of mechanical power generation by leg muscles is substantially higher in old adults [35], assigning a putative role of leg muscle strength in power generation, making it a valid correlate of gait speed, and a target for interventions [14]. Together, in physically highly active healthy old adults, leg muscle strength and leg LTM weakly and similarly correlated with gait performance, favoring neither outcome for predicting mobility.

\section{Relationship between Joint-Specific Leg Muscle Strength and Gait Performance}

At the preferred gait speed, there was some weak but significant evidence for the hypothesis that hip extensor strength only predicted preferred gait speed, whereas only ankle plantarflexion strength predicted old adults' 
maximal gait speed and stride length. The idea of leg strength having a joint-specific relationship with gait performance has emerged from observations that hip and ankle joint torques measured during gait analysis, respectively, increase and decrease [11] and from most [6] but not all data [36], suggesting also a reduction in ankle plantarflexion physiological capacity, including muscle torque in old age. Under our measurement conditions, we found a large reduction in hip but no preferential reductions in ankle plantarflexion function (Table 2), pointing to the importance of total leg output instead of individual joint outputs in gait speed. Indeed, when total leg output was measured on a dynamometer, both age and leg muscle strength contributed independently and in combination with the age-related reorganization of gait mechanics [37].

The significant but poor prediction of gait speed from hip and ankle maximal torque (Table 3, also Fig. $2 \mathrm{~b}$ for summed torques) may reflect that the relative effort, i.e. joint torque during gait relative to maximal available torque, was low in these highly physically active old adults [10], and old adults were able to use a reasonable portion of the available maximal ankle capacity for propulsion generation [38]. Certainly, we found less evidence for ankle function insufficiency, and the pattern of associations between gait speed and joint-specific maximal torques resembled those after strength training using leg press [39]. Our data of hip extensor MIT only predicting stride length at habitual gait speed (29\% explained variance) are in line with hip extensor MIT being best predictor of healthy male old adults' stride length (35\% explained variance) [12]. Further, our finding of ankle plantarflexor MIT only predicting (for both $40 \%$ explained variance) gait speed and stride length at maximal gait speed agrees with MIT of the ankle plantarflexors being the strongest predictor of step length (52\%) at fast gait speed in 70 to
90 -year-old adults [13]. This result would provide support for intervention studies targeting ankle plantarflexor weakness [40]. Indeed, an intervention study focally targeting old adults' ankle muscle function showed some significant improvements in habitual gait speed [40].

While the present study has assets such as measuring joint-specific isokinetic lower-extremity muscle strength, it has a few limitations. First, because of the old participants' high daily physical activity, our results cannot be generalized to old adults suffering from mobility disability. Second, we tested a relatively small number of young $(n=20)$ and old $(n=20)$ adults. Thus, future studies should involve larger sample sizes to enhance statistical power. Third, we used BIA to assess leg LTM representing a proxy of skeletal muscle mass. In future studies, the gold standard (i.e., DEXA) should be used to substantiate the present findings.

\section{Conclusions}

We did not find discriminant relationships between gait performance and leg muscle strength compared with gait performance and leg LTM in healthy young and old adults. Hip extensor strength only predicted habitual gait speed, whereas ankle plantarflexor strength only predicted old adults' maximal gait speed and stride length. The clinical implication of these data is that neither leg muscle strength nor leg LTM favors predicting mobility. Thus, both leg muscle strength and leg LTM should be tested and trained (e.g., strength training to induce muscle hypertrophy) complementarily. Further, strength testing and training of the lower-extremities in old adults should include functional integrated multiarticular rather than monoarticular movements.

\section{References}

1 Abellan van Kan G, Rolland Y, Andrieu S, Bauer J, Beauchet O, Bonnefoy M, Cesari M, Donini LM, Gillette Guyonnet S, Inzitari M: Gait speed at usual pace as a predictor of adverse outcomes in community-dwelling older people an International Academy on Nutrition and Aging (IANA) Task Force. J Nutr Health Aging 2009;13:881-889.

2 Delmonico MJ, Harris TB, Visser M, Park SW, Conroy MB, Velasquez-Mieyer P, Boudreau R, Manini TM, Nevitt M, Newman AB: Longitudinal study of muscle strength, quality, and adipose tissue infiltration. Am J Clin Nutr 2009;90:1579-1585.

Leg Muscle Strength and Mass Correlates of Gait Performance
3 Lexell J: Human aging, muscle mass, and fiber type composition. J Gerontol A Biol Sci Med Sci 1995;50:11-16.

4 Akima $\mathrm{H}$, Kano Y, Enomoto Y, Ishizu M, Okada M, Oishi Y, Katsuta S, Kuno S: Muscle function in 164 men and women aged 20-84 years. Med Sci Sports Exerc 2001;33:220-226.

5 Frontera WR, Hughes VA, Fielding RA, Fiatarone MA, Evans WJ, Roubenoff R: Aging of skeletal muscle: a 12-years longitudinal study. J Appl Physiol (1985) 2000;88:13211326.

6 Danneskiold-Samsoe B, Bartels EM, Bulow PM, Lund H, Stockmarr A, Holm CC, Watjen
I, Appleyard M, Bliddal H: Isokinetic and isometric muscle strength in a healthy population with special reference to age and gender. Acta Physiol (Oxf) 2009;197(suppl 673):1-68.

7 Winegard KJ, Hicks AL, Sale DG, Vandervoort AA: A 12-year follow-up study of ankle muscle function in older adults. J Gerontol A Biol Sci Med Sci 1996;51:B202-B207.

8 Coggan AR, Spina RJ, King DS, Rogers MA, Brown M, Nemeth PM, Holloszy JO: Histochemical and enzymatic comparison of the gastrocnemius muscle of young and elderly men and women. J Gerontol 1992;47:B71B76. 
9 Houmard JA, Weidner ML, Gavigan KE, Tyndall GL, Hickey MS, Alshami A: Fiber type and citrate synthase activity in the human gastrocnemius and vastus lateralis with aging. J Appl Physiol (1985) 1998;85:13371341.

10 Beijersbergen CM, Granacher U, Vandervoort AA, Devita P, Hortobagyi T: The biomechanical mechanism of how strength and power training improves walking speed in old adults remains unknown. Ageing Res Rev 2013;12:618-627.

11 DeVita P, Hortobagyi T: Age causes a redistribution of joint torques and powers during gait. J Appl Physiol 2000;88:1804-1811.

12 Burnfield JM, Josephson KR, Powers CM, Rubenstein LZ: The influence of lower extremity joint torque on gait characteristics in elderly men. Arch Phys Med Rehabil 2000;81:11531157.

13 Judge JO, Davis RB, Ounpuu S: Step length reductions in advanced age: the role of ankle and hip kinetics. J Gerontol A Biol Sci Med Sci 1996;51:M303-M312.

14 Hortobagyi T, Lesinski M, Gabler M, VanSwearingen JM, Malatesta D, Granacher U: Effects of three types of exercise interventions on healthy old adults' gait speed: a systematic review and meta-analysis. Sports Med 2015; 45:1627-1643.

15 Abe T, Ogawa M, Loenneke JP, Thiebaud RS, Loftin M, Mitsukawa N: Relationship between site-specific loss of thigh muscle and gait performance in women: the HIREGASAKI study. Arch Gerontol Geriatr 2012;55:e21e25.

16 Bouchard DR, Heroux M, Janssen I: Association between muscle mass, leg strength, and fat mass with physical function in older adults: influence of age and sex. J Aging Health 2011;23:313-328.

17 SipilaS, Suominen H: Knee extension strength and walking speed in relation to quadriceps muscle composition and training in elderly women. Clin Physiol 1994;14:433-442.

18 Fragala MS, Fukuda DH, Stout JR, Townsend JR, Emerson NS, Boone CH, Beyer KS, Oliveira LP, Hoffman JR: Muscle quality index improves with resistance exercise training in older adults. Exp Gerontol 2014;53:1-6.

19 Francis P, Mc Cormack W, Toomey C, Norton C, Saunders J, Kerin E, Lyons M, Jakeman $\mathrm{P}$ : Twelve weeks' progressive resistance training combined with protein supplementation beyond habitual intakes increases upper leg lean tissue mass, muscle strength and extended gait speed in healthy older women. Biogerontology 2016;1-11.
20 Santos L, Ribeiro AS, Schoenfeld BJ, Nascimento MA, Tomeleri CM, Souza MF, Pina FL, Cyrino ES: The improvement in walking speed induced by resistance training is associated with increased muscular strength but not skeletal muscle mass in older women. Eur J Sport Sci 2017;17:488-494.

21 Ferrucci L, de Cabo R, Knuth ND, Studenski S: Of Greek heroes, wiggling worms, mighty mice, and old body builders. J Gerontol A Biol Sci Med Sci 2012;67:13-16.

22 Ling $\mathrm{CH}$, de Craen AJ, Slagboom PE, Gunn DA, Stokkel MP, Westendorp RG, Maier AB: Accuracy of direct segmental multi-frequency bioimpedance analysis in the assessment of total body and segmental body composition in middle-aged adult population. Clin Nutr 2011;30:610-615.

23 Malavolti M, Mussi C, Poli M, Fantuzzi AL, Salvioli G, Battistini N, Bedogni G: Cross-calibration of eight-polar bioelectrical impedance analysis versus dual-energy X-ray absorptiometry for the assessment of total and appendicular body composition in healthy subjects aged 21-82 years. Ann Hum Biol 2003;30:380-391.

24 Lienhard K, Schneider D, Maffiuletti NA: Validity of the Optogait photoelectric system for the assessment of spatiotemporal gait parameters. Med Eng Phys 2013;35:500-504.

25 Vincent WJ: Statistics in Kinesiology. Champaign, Human Kinetics, 1995.

26 Oberg T, Karsznia A, Oberg K: Basic gait parameters: reference data for normal subjects, 10-79 years of age. J Rehabil Res Dev 1993;30: 210-223.

27 Bohannon RW, Williams AA: Normal walking speed: a descriptive meta-analysis. Physiotherapy 2011;97:182-189.

28 Madsen OR, Lauridsen UB, Hartkopp A, Sorensen $\mathrm{OH}$ : Muscle strength and soft tissue composition as measured by dual energy $\mathrm{x}$ ray absorptiometry in women aged 18-87 years. Eur J Appl Physiol Occup Physiol 1997; 75:239-245.

29 Goodpaster BH, Park SW, Harris TB, Kritchevsky SB, Nevitt M, Schwartz AV, Simonsick EM, Tylavsky FA, Visser M, Newman $\mathrm{AB}$ : The loss of skeletal muscle strength, mass, and quality in older adults: the health, aging and body composition study. J Gerontol A Biol Sci Med Sci 2006;61:1059-1064.

30 Bauer JM, Verlaan S, Bautmans I, Brandt K, Donini LM, Maggio M, McMurdo ME, Mets T, Seal C, Wijers SL: Effects of a vitamin D and leucine-enriched whey protein nutritional supplement on measures of sarcopenia in older adults, the PROVIDE study: a randomized, double-blind, placebo-controlled trial. J Am Med Dir Assoc 2015;16:740-747.
31 Reid KF, Doros G, Clark DJ, Patten C, Carabello RJ, Cloutier GJ, Phillips EM, Krivickas LS, Frontera WR, Fielding RA: Muscle power failure in mobility-limited older adults: preserved single fiber function despite lower whole muscle size, quality and rate of neuromuscular activation. Eur J Appl Physiol 2012; 112:2289-2301.

32 Trombetti A, Reid KF, Hars M, Herrmann FR, Pasha E, Phillips EM, Fielding RA: Ageassociated declines in muscle mass, strength, power, and physical performance: impact on fear of falling and quality of life. Osteoporos Int 2016;27:463-471.

33 Foldvari M, Clark M, Laviolette LC, Bernstein MA, Kaliton D, Castaneda C, Pu CT, Hausdorff JM, Fielding RA, Singh MA: Association of muscle power with functional status in community-dwelling elderly women. J Gerontol A Biol Sci Med Sci 2000;55:M192M199.

34 McNeil CJ, Vandervoort AA, Rice CL: Peripheral impairments cause a progressive agerelated loss of strength and velocity-dependent power in the dorsiflexors. J Appl Physiol (1985) 2007;102:1962-1968.

35 Yamauchi J, Mishima C, Nakayama S, Ishii N: Aging-related differences in maximum force, unloaded velocity and power of human leg multi-joint movement. Gerontology 2010;56: 167-174.

36 Harbo T, Brincks J, Andersen H: Maximal isokinetic and isometric muscle strength of major muscle groups related to age, body mass, height, and sex in 178 healthy subjects. Eur J Appl Physiol 2012;112:267-275.

37 Hortobagyi T, Rider P, Gruber AH, DeVita P: Age and muscle strength mediate the age-related biomechanical plasticity of gait. Eur J Appl Physiol 2016;116:805-814.

38 Franz JR: The age-associated reduction in propulsive power generation in walking. Exerc Sport Sci Rev 2016;44:129-136.

39 Uematsu A, Tsuchiya K, Kadono N, Kobayashi H, Kaetsu T, Hortobagyi T, Suzuki S: A behavioral mechanism of how increases in leg strength improve old adults' gait speed. PLoS One 2014;9:e110350.

40 Hartmann A, Murer K, de Bie RA, de Bruin ED: The effect of a foot gymnastic exercise programme on gait performance in older adults: a randomised controlled trial. Disabil Rehabil 2009;31:2101-2110. 